\title{
TRADUÇÃO E VALIDAÇÃO DO QUESTIONÁRIO DE AVALIAÇÃO DE GRAVIDADE DOS SINTOMAS E DO ESTADO FUNCIONAL NA SÍNDROME DO TÚNEL DO CARPO
}

\author{
Carmelinda Correia de Campos ${ }^{1}$, Gilberto Mastrocola Manzano², \\ Lucilia Bannwart de Andrade ${ }^{3}$, Adauto Castelo Filho4, João Antonio Maciel Nóbrega ${ }^{5}$
}

\begin{abstract}
RESUMO - Este estudo tem por objetivo traduzir, fazer a equivalência cultural e validar o questionário de Levine et al. (1993) ou Boston Carpal Tunnel Questionnaire (BCTQ), para o português. O BCTQ aplicado a pacientes selecionados pela pergunta "Qual o motivo que a(o) levou a procurar o médico e que fez com que ele solicitasse este exame?" mostrou boa reprodutibilidade. A validade foi medida comparando o escore de gravidade dos sintomas (EGS) e o escore do estado funcional (EEF) aos resultados dos testes de força de preensão e pinça, Minnesota, discriminação entre dois pontos, Semmes-Weinstein e velocidade de condução sensitiva no nervo mediano. A consistência interna foi avaliada pelo coeficiente $\alpha$ de Chronbach, comparando o EGS ao EEF. A propriedade de medida foi avaliada pelo teste t pareado, comparando escores pré-cirúrgicos aos pós-cirúrgicos. Reprodutibilidade, consistência interna, validade e propriedades de medida do BCTQ traduzido foram semelhantes às encontradas por Levine et al. na versão original.
\end{abstract}

PALAVRAS-CHAVE: questionário de Boston, síndrome do túnel do carpo, condução nervosa.

\begin{abstract}
Translation and validation of an instrument for evaluation of severity of symptoms and the functional status in carpal tunnel syndrome

ABSTRACT - The objective of the present study was to translate, to do cultural equivalence and validation of the Levine et al. (1993) or Boston Carpal Tunnel Questionnaire (BCTQ) to Portuguese. The BCTQ application to patients, selected by the question "What was the reason that brought you to look for a physician and that led him to ask this examination?" showed very good reproducibility. The validity was measured through the comparison of the severity symptoms scores (SSS) and the functional status scores (FSS) with the results of grip forces, Minnesota, two point discrimination, Semmes-Weinstein filaments perception and sensory conduction at the median nerve. The internal consistency was evaluated through Chronbach's alpha coefficient comparing the SSS and the FSS. The measuring properties were evaluated through paired t-test between pre and pos-surgical scores. Reproducibility, internal consistency, validation and measuring properties of the translated BCTQ were similar to those found by Levine et al. with the original version.
\end{abstract}

KEY WORDS: Boston carpal tunnel questionnaire, carpal tunnel syndrome, nerve conduction.

Significativo número de pacientes com queixas de parestesias e/ou dores nas mãos e/ou punhos é diariamente encaminhado para avaliação eletrofisiológica. Com frequência, essas queixas fazem parte de um conjunto de sinais e sintomas conhecido como síndrome do túnel carpo (STC) ${ }^{1}$, sendo assim denominado porque a compressão do nervo mediano no carpo (CNMC) é sua causa mais comum ${ }^{2,3}$. Tais sin- tomas, no entanto, podem ter diversas causas ${ }^{4-8}$, porém, por sua frequência e suas implicações sócioeconômicas, CNMC é, sem dúvida, a causa mais importante?

Embora as parestesias sejam consideradas indicativas de comprometimento dos nervos periféricos, elas podem, com frequência, ser encontradas em pacientes com alterações osteoarticulares e musculoes-

Estudo realizado no Setor de Neurofisiologia Clínica da Disciplina de Neurologia da Universidade Federal de São Paulo - Escola Paulista de Medicina (UNIFESP-EPM), São Paulo SP, Brasil: 'Pós-Graduanda do Setor de Neurofisiologia Clínica da Disciplina de Neurologia; ${ }^{2}$ Professor Afiliado da Disciplina de Neurologia; ${ }^{3}$ Pós-Graduanda da Disciplina de Reabilitação; ${ }^{4}$ Professor Adjunto da Disciplina de DIPO; ${ }^{5}$ Professor Adjunto da Disciplina de Neurologia.

Recebido 16 Abril 2002, recebido na forma final 20 Julho 2002. Aceito 9 Agosto 2002. 
queléticas ${ }^{10,11}$. Entre os pacientes com essas queixas estão incluídos trabalhadores da indústria, nos quais a incidência de CNMC e de alterações ósteo-articulares e músculo esqueléticas é tida como maior do que na população geral ${ }^{10}$. Por isso, a identificação da presença ou ausência de CNMC nesse grupo tem especial interesse, não só pela conduta terapêutica mas também por suas implicações médico-trabalhistas ${ }^{9}$. Nos últimos anos, observa-se interesse crescente por parte das seguradoras, empregadores, órgãos públicos e pacientes, em saber se certos tratamentos específicos, especialmente cirúrgicos, satisfazem as necessidades dos que a eles se submetem. Por isso, a comunidade médico-científica tem procurado desenvolver estudos que avaliem os resultados de tratamentos. Diferentes tipos de instrumentos têm sido propostos ${ }^{12,13}$, entre eles os questionários $^{9,13,14}$, sendo alguns específicos para doenças comuns e alguns abordando não só as respostas destas aos tratamentos, mas também os aspectos emocionas e sociais envolvidos e como tais tratamentos interferem na qualidade de vida dos que os recebem. Um exemplo é o SF 36, já traduzido para vários idiomas ${ }^{15}$.

A utilização de questionários como parâmetro de avaliação é útil, por permitir a padronização, a uniformização e a reprodutibilidade das medidas a que se propõe ${ }^{9,13-17}$. No entanto, a escolha de um instrumento de avaliação deve levar em conta se seus componentes são claros, se ele é simples, de fácil compreensão e aplicação, e se possui tempo de administração apropriado ${ }^{18}$. Quando é elaborado um questionário, suas propriedades de medida precisam ser testadas e validadas primeiro num grupo de pacientes, para que posteriormente possam ser utilizadas em estudos populacionais ${ }^{15}$. Em 1993, foi desenvolvido por Levine et al. ${ }^{9}$ um questionário que foi denominado Boston Carpal Tunnel Questionnaire (BCTQ) por Padua et al. ${ }^{5}$, para ser aplicado a pacientes com síndrome do túnel do carpo, com a finalidade de avaliar a gravidade dos sintomas e o grau de desabilidade manual. Este instrumento de avaliação foi reconhecido como reprodutível, válido, com consistência interna e capaz de responder a mudanças clinicas. Como este instrumento baseia-se na quantificação da parestesia e dor nas mãos e punhos, entre outros sintomas, nos propusemos a traduzi-lo e validá-lo para o Brasil.

O objetivo deste estudo foi traduzir, fazer uma adaptação trans-cultural, validar e testar as propriedades de medida do instrumento de avaliação proposto por Levine et al. ${ }^{9}$, denominado: "Selfadministered questionnaire for the assessment of severity of symptoms and functional status in carpal tunnel syndrome".

\section{MÉTODO}

A pesquisa foi aprovada pelo Comitê de Ética em Pesquisa da Universidade Federal de São Paulo (UNIFESP), protocolo $\mathrm{n}^{\circ} 721 / 99$.

1) Tradução - a) o BCTQ foi traduzido do inglês para o português por dois professores de inglês cientes da finalidade; b) as duas traduções foram comparadas e adaptadas, dando origem à versão 1 ; c) a versão 1 foi traduzida do português para o inglês, por dois professores de língua nativa inglesa, não cientes da finalidade; d) as versões foram comparadas com o original e as discrepâncias foram corrigidas, dando origem à versão 2 .

2) Equivalência cultural - a) a cada uma das questões da versão 2 foi acrescentada a opção "não aplicável", com a intenção de identificar as não compatíveis, não compreendidas ou não executadas pela nossa população; b) a versão 2 foi aplicada a 20 pacientes com parestesias e/ou dores nas mãos e punhos, selecionados aleatoriamente, para que as questões com mais de $15 \%$ de respostas "não aplicável" fossem substituídas por outras com mesmo conceito, dando origem à versão definitiva.

3) Reprodutibilidade - A versão definitiva do BCTQ (anexo) foi aplicada aos primeiros 50 pacientes com queixas de parestesias e/ou dores nas mãos e/ou punhos, selecionados pela pergunta: "Qual o motivo que a(o) levou a procurar o médico e que fez com ele solicitasse este exame?" Na primeira consulta, o questionário foi aplicado por dois entrevistadores, e após um período de no máximo 15 dias, foi reaplicado por um dos entrevistadores (CCC). A reprodutibilidade inter e intra examinadores foi comparada pelo coeficiente Kappa. Todos os pacientes foram também submetidos à avaliação eletrofisiológica, seguindo o protocolo descrito previamente ${ }^{19}$.

4) Consistência Interna - O coeficiente $\alpha$ de Chronbach foi calculado para o conjunto de questões referentes à gravidade dos sintomas e para o conjunto referente ao estado funcional, utilizando-se as respostas ao questionário obtidas dos pacientes avaliados para a reprodutibilidade.

5) Validade - A validade do BCTQ foi testada, num grupo de 23 pacientes, através do coeficiente de correlação de Spearman, comparando-se os escores obtidos para a gravidade dos sintomas (EGS) e para o estado funcional (EEF) aos resultados de outros testes: de força de preensão e pinça, habilidade manual de Minnesota, discriminação entre dois pontos, dos monofilamentos de Semmes-Weinstein 
e da velocidade de condução sensitiva no nervo mediano. Para obtenção dos escores (EGS e EEF), foram somados os pontos (1 a 5) obtidos na resposta de cada pergunta e a somatória foi dividida pelo número de respostas. As questões não respondidas foram excluídas do cálculo.

6) Propriedade de medida - Os escores pré-cirúrgicos foram comparados, pelo teste $t$ pareado, aos escores pós-cirúrgicos de 17 pacientes submetidos à cirurgia de descompressão do nervo mediano no punho.

\section{RESULTADOS}

A tradução e a equivalência cultural - não ofereceram dificuldades, por tratar-se de questionário simples, com questões abordando sintomas habituais e atividades corriqueiramente executadas pela nossa população. Não foi necessário alterar nenhuma das perguntas, para a equivalência cultural, após o estabelecimento da versão 2 que ficou sendo a versão definitiva (Tabela 1 A e B).

Reprodutibilidade - a avaliação das respostas mostrou que o questionário teve boa reprodutibilidade, considerando-se os critérios de Landis e $\mathrm{Koch}^{20}$, tanto inter-entrevistadores (o coeficiente Kappa variou de 0,42 a 0,84 , com a maioria dos coeficientes acima de 0,60 ) quanto intra-entrevistador (o coeficiente Kappa variou de 0,35 a 0,84, com a maioria dos coeficientes acima de 0,55 e apenas um coeficiente abaixo de 0,40 ).

Consistência interna - o coeficiente $\alpha$ de Chronbach foi de 0,83 para a escala de gravidade de sintomas e de 0,90 para o estado funcional, mostrando boa consistência interna.

Validade - avaliada pelo coeficiente de correlação de Spearman, mostrou que: a) os EGS e os EEF tiveram alta correlação com os testes de força de preensão: (EGS: $r_{s}=-0,56, p=0,0003$ e EEF: $r_{s}=-0,69$, $p=0,0000)$ e pinça: (EGS: $r_{s}=-0,53, p=0,001$ e EEF: $\left.r_{s}=-0,67 p=0,0000\right)$. b) o EEF mostrou alta correlação $\left(r_{s=} 0,57, p=0,0003\right)$ com o teste de habilidade manual de Minnesota e o EGS mostrou correlação limítrofe $\left(r_{s}=0,33, p=0,048\right)$. c) os EGS e os EEF não mostraram correlação com os testes de discriminação entre dois pontos (EGS: $r_{s}=0,14, p=0,399$ e EEF: $r_{s}=0,14, p=0,413$ ), de monofilamentos (EGS: $r_{s}=$ $0.04, p=0,821$ e EEF: $\left.r_{s}=-0,30, p=0,073\right)$ e com medidas de velocidade de condução sensitiva (EGS: $r_{s}$ $=0,149, p=0,386$ e EEF: $r_{s}=-0,081, p=0,637$ )

Propriedade de medida - foi avaliada comparando-se os escores pré-cirúrgicos e pós-cirúrgicos, pelo teste $t$ pareado. Observamos diferença significante entre os escores pré e pós cirúrgicos (EGS pré / EGS pós $t=6,238-g l 14-p<0,0001$ ) (EEF pré / EEF pós $t=3,490 \mathrm{gl} 14-p<0,0036)$

\section{DISCUSSÃO}

Em estudo prévio ${ }^{19}$, observamos que pacientes com queixas de parestesias e/ou dores nas mãos e/ ou punhos são frequentes entre os encaminhados para exame eletrofisiológico. A partir dessa constatação, resolvemos tentar definir melhor a sintomatologia desse grupo de pacientes, através da aplicação do BCTQ. Com essa finalidade selecionamos aleatoriamente 50 pacientes com queixas de parestesias e/ou dores nas mãos e/ou punhos, através da pergunta: "Qual o motivo que o(a) levou a procurar o médico e que fez com que ele solicitasse este exame?" e a eles aplicamos o questionário devidamente traduzido e adaptado para o nosso meio.

Escolhemos o questionário desenvolvido por Levine et al. ${ }^{9}$ (BCTQ) por ser simples, de fácil compreensão e aplicação, e com tempo de administração apropriado. Também foi escolhido por já estar sendo utilizado por outros pesquisadores ${ }^{5,8,21,22}$ e ter se mostrado útil na avaliação da gravidade dos sintomas e do estado funcional em pacientes portadores de CNMC. Porém, como ocorre com a maioria dos instrumentos de avaliação, ele foi formulado na língua inglesa e, portanto, direcionado a população com idioma e cultura diferentes dos nossos. Por isso, para que pudesse ser utilizado na nossa população, foi necessário não só traduzi-lo rigorosamente, mas também submetê-lo a adaptação transcultural.

Depois de traduzido, ele foi testado quanto à sua reprodutibilidade, consistência interna, validade e propriedades de medida, seguindo as regras que foram propostas por Guillemin ${ }^{23}$. Nossos resultados foram semelhantes aos encontrados por Levine et al. ${ }^{9}$, embora esse trabalho difira do nosso por ter sido aplicado retrospectivamente a 36 pacientes operados de CNMC há mais de 1 ano e prospectivamente no pré e pós-operatório de 26 pacientes com CNMC, com a finalidade de avaliar o resultado cirúrgico.

O questionário, depois de traduzido, mostrou-se semelhante ao original de Levine et al. ${ }^{9}$ quanto à reprodutibilidade, consistência interna e sensibilidade para detectar mudanças clínicas. As comparações dos EGS e dos EEF com testes que medem a força de preensão e pinça mostraram alta correlação, na nossa avaliação, e moderada correlação na de Levine et al. ${ }^{9}$. Também encontramos alta correlação entre os EEF e o teste de Minnesota, o que não foi testado no trabalho de Levine et al. ${ }^{9}$. 
Tabela 1A. Questionário de Levine et al. (1993)9.

As questões a seguir referem-se aos seus sintomas, num período típico de 24 horas, durante as duas últimas semanas (assinale uma resposta para cada pergunta).

1) Qual a intensidade da dor na mão ou no punho que você sente à noite?
1 - eu não tenho dor na mão ou no punho à noite.
4 - dor intensa
2 - pouca dor
5 - dor muito intensa
3 - dor moderada

2) Quantas vezes a dor na mão ou no punho acordou você durante uma noite típica, nas duas últimas semanas?
1 - nenhuma
4 - quatro a cinco vezes
2 - uma
5 - mais que cinco vezes.

3 - duas a três vezes

3) Você usualmente sente dor na mão ou no punho durante o dia?

1 - eu nunca tenho dor durante o dia

4 - eu tenho dor intensa durante o dia

2 - eu tenho pouca dor durante o dia

5 - eu tenho dor muito intensa durante o dia

3 - eu tenho dor moderada durante o dia

4) Com que frequência você sente dor na mão ou no punho durante o dia?
1 - nunca
4 - mais que cinco vezes por dia
2 - uma ou duas vezes por dia
5 - a dor é constante

3 - três a cinco vezes por dia

5) Quanto tempo em média duram os episódios de dor durante o dia?
1 - eu nunca tenho dor durante o dia
4 - mais que 60 minutos
2 - menos que 10 minutos
5 - a dor é constante durante o dia

3 - de 10 a 60 minutos

6) Você tem adormecimento ( perda da sensibilidade) em sua mão?
1 - não
2 - eu tenho pouco adormecimento
3 - eu tenho adormecimento moderado

4 - eu tenho adormecimento intenso

5 - eu tenho adormecimento muito intenso

7) Você tem fraqueza na sua mão ou punho?
1 - sem fraqueza
2 - pouca fraqueza
3 - fraqueza moderada

4 - fraqueza intensa
5 - fraqueza muito intensa

8) Você tem sensação de formigamento em sua mão?
1 - sem formigamento
2 - pouco formigamento
3 - formigamento moderado

4 - formigamento intenso

5 - formigamento muito intenso

9) Qual a intensidade do adormecimento (perda da sensibilidade) ou do formigamento à noite?
1 - eu não tenho adormecimento ou formigamento à noite
4 - intensa
2 - pouca
5 - muito intensa

3 - moderada

10) Com que frequência o adormecimento ou o formigamento acordaram você durante uma noite típica, nas duas últimas semanas?
1 - nenhuma
2 - uma
4 - quatro a cinco vezes
3 - duas a três vezes
5 - mais que cinco vezes

11) Você tem dificuldade em pegar e usar pequenos objetos, tais como chaves ou canetas?
1 - sem dificuldade
2 - pouca dificuldade
3 - dificuldade moderada
4 - dificuldade intensa
5 - dificuldade muito intensa

Levine et al. ${ }^{9}$ observaram que os testes dos monofilamentos, os de duplo estímulo e das velocidades de condução sensitiva tiveram fraca correlação com os EGS e os EEF. O mesmo foi observado na nossa avaliação. É possível que a falta de correlação significante entre EGS e EEF e as medidas da condução sensitiva observada nos nossos resultados, assim como nos de Levine et al. ${ }^{9}$, seja decorrente do fato dessas duas técnicas medirem diferentes aspectos da mesma afecção. Nosso achado, assim como o de Levine et al. ${ }^{9}$, foi diferente do de You et al. ${ }^{8}$, que referiu ter encontrado boa correlação entre EGS e as medidas de condução.

O questionário proposto por Levine et al. ${ }^{9}$ já vem sendo utilizado por autores de língua inglesa ${ }^{8,21,22} \mathrm{e}$ já foi traduzido para a língua italiana ${ }^{5}$, sendo aplicado em estudos de pacientes com STC. A tradução e validação para a língua portuguesa permitem que ele passe a ser utilizado na avaliação da nossa população, com a vantagem de obtermos resultados uniformes, padronizados e reprodutíveis, facilitando assim a comparação dos sintomas e das desabili- 
Tabela 1B. Num dia típico, durante as duas últimas semanas, os sintomas da sua mão ou punho têm-Ihe causado alguma dificuldade em fazer as atividades listadas abaixo?

\begin{tabular}{|c|c|c|c|c|}
\hline \multicolumn{5}{|l|}{ Atividade } \\
\hline Escrever & 1 & 2 & 3 & 4 \\
\hline Abotoar as roupas & 1 & 2 & 3 & 4 \\
\hline Segurar um livro enquanto lê & 1 & 2 & 3 & 4 \\
\hline Segurar o telefone & 1 & 2 & 3 & 4 \\
\hline Abrir a tampa de um vidro & 1 & 2 & 3 & 4 \\
\hline Trabalhos domésticos & 1 & 2 & 3 & 4 \\
\hline Carregar sacos do supermercado & 1 & 2 & 3 & 4 \\
\hline Tomar banho e vestir-se & 1 & 2 & 3 & 4 \\
\hline Nenhuma dificuldade & 1 & & & \\
\hline Pouca dificuldade & 2 & & & \\
\hline Dificuldade moderada & 3 & & & \\
\hline Dificuldade intensa & 4 & & & \\
\hline $\begin{array}{l}\text { Não realiza a atividade de jeito nenhum } \\
\text { por causa dos sintomas nas mãos e punhos }\end{array}$ & 5 & & & \\
\hline
\end{tabular}

\section{dades manuais em futuras pesquisas científicas e em ensaios clínicos e terapêuticos nos quais venha a ser utilizado, como, por exemplo, nos pacientes porta- dores de parestesias e/ou dores nas mãos e/ou pu- nhos, causadas por compressão do nervo mediano no carpo ou por outras doenças.}

\section{REFERÊNCIAS}

1. Phalen GS. The carpal tunnel syndrome: seventeen years' experience in diagnosis and treatment of six hundred fifty-four hands. J Bone Joint Surg 1966;48A:211-228.

2. Dawson DM, Hallett M. Entrapment neuropathies. 3.Ed., Philadelphia: Lippincott Raven 1999:20-94.

3. Kimura J. Electrodiagnosis in diseases of nerve and muscle. 2.Ed., Philadelphia: FA Davis 1989:501-504

4. American Association of Electrodiagnostic Medicine, American Academy of Neurology, American Academy of Physical Medicine Rehabilitation. Literature review of the usefulness of nerve conduction studies and electromyography for evaluation of patients with carpal tunnel syndrome. Muscle Nerve 1993;16:1392-1414.

5. Padua L, Padua R, Aprile I, Tonali P. Italian multicenter study of carpal tunnel syndrome differences in the clinical and neurophysiological features between male and female patients. J Hand Surg 1999; 24B: 579-582.

6. Quality Standards Subcommittee of the American Academy of Neurology: practice parameter for carpal tunnel syndrome. Neurology 1993;43:2406-2409.

7. Stevens JC. AAEE minimonograph: the electrodiagnosis of carpal tunnel syndrome. Muscle Nerve 1997;20:1477-1486.

8. You H, Simmons Z, Freivalds A, Kothari MJ, Naidu SH. Relationships between clinical symptom severity scales and nerve conduction measures in carpal tunnel syndrome. Muscle Nerve 1999;22:497-501.

9. Levine DW, Simmons BP, Koris MJ, et al. A self-administered questionnaire for the assessment of severity of symptoms and functional status in carpal tunnel syndrome. J Bone Joint Surg 1993;75A:1585-1592.
10. Lazaro RP. Neuropathic symptoms and musculoskeletal pain in carpal tunnel syndrome: prognostic and therapeutic implications. Surg Neurol 1997;47:115-119.

11. Mackinnons E, Novak CB. Pathogenesis of cumulative trauma disorder. J Hand Surg 1994;19A:873-883.

12. Katz N, Stirrat CR. A self-administered hand diagram for the diagnosis of carpal tunnel syndrome. J Hand Surg 1990;15:360-363.

13. Stevens JC, Smith BE, Weaver AL, Bosch EP, Deen HG, Wilkens JA. Symptoms of 100 patients with electromyographically verified carpal tunnel syndrome. Muscle Nerve 1999;22:1448-1456.

14. Franzblau A, Salermo DF, Armstrong TJ, Werner RA. Test-retest reliability of an upper- extremity discomfort questionnaire in an industrial population. Scand J Work Environ Health 1997;23:299-307.

15. Ciconelli RM. Tradução para o português e validação do questionário genérico de avaliação de qualidade de vida "medical outcomes study 36 item short-form health survey (SF36). Tese de doutorado: Universidade Federal de São Paulo, São Paulo, 1997.

16. Ferry S, Alam JS, T Pritchard, Janet K, Peter C.The association between different patterns of hand symptoms and objective evidence of median nerve compression. Arthritis Rheum 1998;41:720-724.

17. Homan M, Franzblau A, Werner RA. Albers JW, Armstrong TJ, Bromberg MB. Agreement between symptom surveys, physical examination procedures and electrodiagnostic findings for the carpal tunnel syndrome. Scand J Work Environ Health 1999; 25:115-124.

18. Belll MJ, Bombardier C, Tugwell P. Measurement of functional status, quality of life and utility in rheumatoid arthritis. Arthritis Rheum 1990;33:591-601.

19. Campos CC, Manzano GM, Castelo AF, Nóbrega JAM. Parestesias e/ ou dores nas mãos e/ou punhos como motivo de encaminhamento para estudo eletroneuromiográfico. Arq Neuropsiquiatr (in press)

20. Landis JR, Koch GG. The measurement of observer agreement for categorical data. Biometrics 1977;33:159-174.

21. Atrosshi I, Breidenbach WC, McCabe SJ, Louisville KY. Assessment of the carpal tunnel in patients with nerve-compression symptoms. J Hand Surg 1997; 22A:222-227.

22. Besset L, Keller RB, Liang MH, Simmons BP, Fossel AH, Katz JN. Patient's preferences and their relationship with satisfaction following carpal tunnel release. J Hand Surg 1997;22A:613- 619.

23. Guillemin F. Cross-cultural adaptation and validation of Health Status Measures. Scand J Rheumatol 1995;24:61-63. 